International Journal of Innovative Engineering Applications

Journal homepage: https://dergipark.org.tr/ijiea

\title{
THE GaN EPILAYER GROWN BY MOVPE: EFFECT OF THE DIFFERENT NUCLEATION LAYER TEMPERATURES
}

\author{
Ismail Altuntaş ${ }^{* 1,2}$ iD, Sezai Elagöz ${ }^{3}$ iD \\ ${ }^{I}$ Department of Nanotechnology Engineering, Sivas Cumhuriyet University, 58140 Sivas, Turkey \\ ${ }^{2}$ Nanophotonics Research and Application Center, Sivas Cumhuriyet University, 58140, Sivas, Turkey \\ ${ }^{3}$ ASELSAN-Microelectronics, Guidance and Electro-Optics, Ankara, Turkey
}

\begin{abstract}
Original scientific paper

Effect of different nucleation layer temperatures (LT-GaN growth temperature) on the properties of the subsequent GaN epilayer grown by MOVPE is investigated. In-situ reflectance curves demonstrate that higher LT-GaN growth temperatures cause fast coalescence (shorter transition time) of GaN nucleation islands. Both photoluminescence (PL) and high-resolution x-ray diffraction (HRXRD) are used to demonstrate the influence of LT-GaN growth temperature on optical and structural properties of subsequent GaN epilayer, respectively. It is observed that the change of LT-GaN growth temperature has an effect on both full-width at half-maximum (FWHM) values obtained from the results of HRXRD measurement and yellow luminescence peak intensity. It is seen that the yellow luminescence peak intensities for samples alter with LT-GaN growth temperature.
\end{abstract}

Keywords: Characterization, epitaxy, gallium nitride, metal organic vapor phase epitaxy, XRD.

\section{Introduction}

The III-Nitride based semiconductors have attracted great attention for recent years because of many important application areas in technological devices [1]. Their application areas are listed as high electron mobility transistors (HEMTs), solar-blind detectors, detectors of ionizing radiation and scintillators, UV emitters for purification, curing and disinfection, light-emitting, and lasers diodes [2-11]. Especially, the GaN grown via Metal Organic Vapour Phase Epitaxy (MOVPE) is the most popular of the III-Nitride based semiconductors (AIN, InN, and their alloys) [12]. The GaN substrate is not in use for some reasons such as not large enough size and inexpensive to compete with the other competitors such as sapphire and silicon. Many studies to achieve heteroepitaxial GaN epilayer grown on foreign substrates have been done by using MOVPE [13-15]. In the case of using a different substrate, the quality of the GaN layer is affected due to thermal expansion difference and large lattice mismatch between the used substrate and the GaN layer [16]. Sapphire substrate is commonly used to grow GaN epilayer. Nakamura et al. have used a two-steps growth process (first step low-temperature and second step high temperature) to improve the quality of the GaN epilayers [12]. For this reason, some parameters affect $\mathrm{GaN}$ quality during both LT GaN and HT-GaN growth. Many MOVPE studies have been shown the impact of growth parameters such as growth temperature, reactor pressure, annealing, growth rate, total carrier gas flow, V/III ratio (ratio between the partial pressures of $\mathrm{V}$ and III elements in the gas phase), etc. in both LT-GaN and HT-GaN growth [17-22]. M. S. Yi et al. have focused on the structure of the $\mathrm{GaN}$ nucleation layers to investigate the effect of the growth temperature. They have demonstrated that both the stacking order and the order in the atomic positions in a $\mathrm{GaN}$ atomic epilayer significantly changed with changing the set growth temperature. [22]. AlN layer has been used as a nucleation layer instead of LT-GaN layer in previous studies. D. D. Koleske et al. have shown the influence of the temperature of the AlN nucleation layer on electronic properties of the subsequent $\mathrm{GaN}$ epilayer. The growth temperature affects AlN morphology because temperature influenced the formation of $\mathrm{GaN}$ epilayer and electron mobility [23].

The present study aims to investigate the influence of growth temperature of the LT-GaN layer on structural and optical characteristics of subsequent GaN layer grown via MOVPE. Characterization equipment such as HRXRD and $\mathrm{PL}$ are used to analyze the change of the properties such as structural and optical of GaN epilayer with the change of used growth temperature in LT-GaN growth. It has shown that different LT-GaN growth temperatures change the ratio of luminescence intensities of the near-band gap transition and the yellow transition. It is seen that there is a relation between XRD results and LT-GaN growth temperature. According to FWHM values obtained from symmetrical and asymmetrical scans, the optimum LT- 
$\mathrm{GaN}$ growth temperature can be used to obtain a $\mathrm{GaN}$ crystal with a lower FHWM value. Besides, the effect of LT-GaN growth temperature on the growth rate and coalescence time is observed.

\section{Experimental}

All films have been grown on a 2-inches sapphire substrate via a horizontal flow MOVPE system with AIXTRON 200/4 RF-S model. The system includes Luxtron $880 \mathrm{~nm}$ reflectometer to take valuable information such as growth rate per second ( $\mathrm{nm} / \mathrm{s})$, surface quality, and an optical fiber thermometry-light-pipe to measure growth temperature during the growth process. The high-purity ammonia $\left(\mathrm{NH}_{3}\right)$, Trimethylgallium (TMGa, $\left.\mathrm{Ga}\left(\mathrm{CH}_{3}\right)_{3}\right)$, and hydrogen $\left(\mathrm{H}_{2}\right)$ have been used as the source precursors for $\mathrm{N}$ and $\mathrm{Ga}$ and carrier gas, respectively. Before growth, the substrate has been heated at a higher temperature compared to HT-GaN growth temperature and thermally desorbed in $\mathrm{H}_{2}$ ambient for $10 \mathrm{~min}$ to remove possible contaminants like surface impurities. The reactor temperature has been decreased to LT-GaN growth temperature value and $20 \mathrm{~s} \mathrm{NH}_{3}$ flow has been supplied to the surface of the sapphire substrate for the nitridation process. LT-GaN layer called a nucleation layer has been grown at 200 mbar and different temperature values. Then, the substrate temperature has been raised for the HT-GaN epitaxial layer deposition under the $\mathrm{NH}_{3}$ ambient. The reactor temperature has been fixed at this temperature to initiate the recrystallization of the $\mathrm{GaN}$ nucleation layer. After recrystallization, the GaN layer has been grown to have Fabry-Perot oscillations at the HT-GaN growth temperature. Table 1 gives the LT-GaN growth temperatures for 4 samples that have the same growth conditions. Several characterization techniques like in-situ reflectance measurement, HRXRD, and PL have been employed to understand the effect of LT-GaN growth temperature on the properties of GaN. HRXRD measurements have been conducted to obtain the FWHM values of omega scans for symmetric (00.2) plane and asymmetric (10.2) plane reflections of the GaN epilayer. The PL measurements have been done with the $325 \mathrm{~nm} \mathrm{He}$ $\mathrm{Cd}$ laser as an exciting source. The information about the materials and devices during the obtaining of the $\mathrm{GaN}$ epilayer has been given in Table 2 .

Table 1. LT-GaN growth temperature of each sample

\begin{tabular}{cc}
\hline Sample Name & LT-GaN Growth Temperature $\left({ }^{0} \mathbf{C}\right)$ \\
\hline A & 455 \\
B & 495 \\
C & 525 \\
D & 550 \\
\hline
\end{tabular}

Table 2. The list using materials and devices to grown GaN epilayer

\begin{tabular}{|c|c|c|c|}
\hline Type & Name & Aim & Source \\
\hline Material & Sapphire & $\begin{array}{c}\text { Used as a substrate to grow the } \\
\text { GaN layer }\end{array}$ & imported from abroad \\
\hline Material & high-purity ammonia $\left(\mathrm{NH}_{3}\right)$ & $\begin{array}{c}\text { Used source precursor to } \\
\text { obtained for } \mathrm{N}\end{array}$ & imported from abroad \\
\hline Material & trimethylgallium $(\mathrm{TMGa}$, & $\begin{array}{c}\text { Used source precursor to } \\
\text { obtained for Ga }\end{array}$ & imported from abroad \\
\hline Material & hydrogen $\left(\mathrm{H}_{2}\right)$ & $\begin{array}{c}\text { Used as a carrier gas and an } \\
\text { ambient }\end{array}$ & supplied domestically \\
\hline Device & $\begin{array}{c}\text { Metal Organic Chemical Vapour } \\
\text { Phase Epitaxy }\end{array}$ & $\begin{array}{c}\text { Used during the growth of GaN } \\
\text { epilayer }\end{array}$ & $\begin{array}{c}\text { Provided by CUNAM } \\
\text { (AIXTRON 200/4 RF-S) }\end{array}$ \\
\hline Device & $\begin{array}{c}\text { High Resolution X-Ray } \\
\text { Diffraction }\end{array}$ & $\begin{array}{c}\text { Used to obtain structural } \\
\text { characterization }\end{array}$ & $\begin{array}{c}\text { Provided by CÜNAM } \\
\text { (Rigaku SmartLab) }\end{array}$ \\
\hline Device & Photoluminesence & $\begin{array}{c}\text { Used to obtain optical } \\
\text { characterization }\end{array}$ & $\begin{array}{c}\text { Provided by ERMAKSAN } \\
\text { (PL mapper) }\end{array}$ \\
\hline
\end{tabular}

\section{The Research Findings and Discussion}

One of the most important measurement techniques for MOVPE growths is in-situ reflectance measurement which gives valuable information such as growth rate, surface situation, etc. at the same time [24]. Detailed information about the growth steps of $\mathrm{GaN}$ is mentioned elsewhere [18, 25]. Figure 1 (a) demonstrates the optical reflectance curves of Samples A-D. The starting points of in-situ reflectance curves of samples have been fixed and separated for better visibility and comparison. The effect of LT-GaN growth temperature on the growth rate of the nucleation layer is shown in Figure 1 (b). It is clear that when LT-GaN growth temperature is increased, the growth rate during the growth of the nucleation layer increases.
The intensity of reflection obtained from the surface of the nucleation layer via Luxtron $880 \mathrm{~nm}$ laser is proportional to the thickness of the nucleation layer. The reflection heights for each sample are adjusted at the same height to fix the thickness of the nucleation layer. Thus, it has been assumed that all nucleation layers have the same thickness.

Recovery time $(\Delta t)$ which corresponds to the duration of optical reflectance intensity from the recrystallization to the first Fabry-Perot oscillation is found for each sample. The effect of LT-GaN growth temperature on the recovery time is revealed in Figure 2. It has been found that the recovery time decreases with the higher growth temperature of the nucleation layer. The temperature difference between LT-GaN and HT-GaN growth is different for every sample. It is believed that larger $\mathrm{GaN}$ 

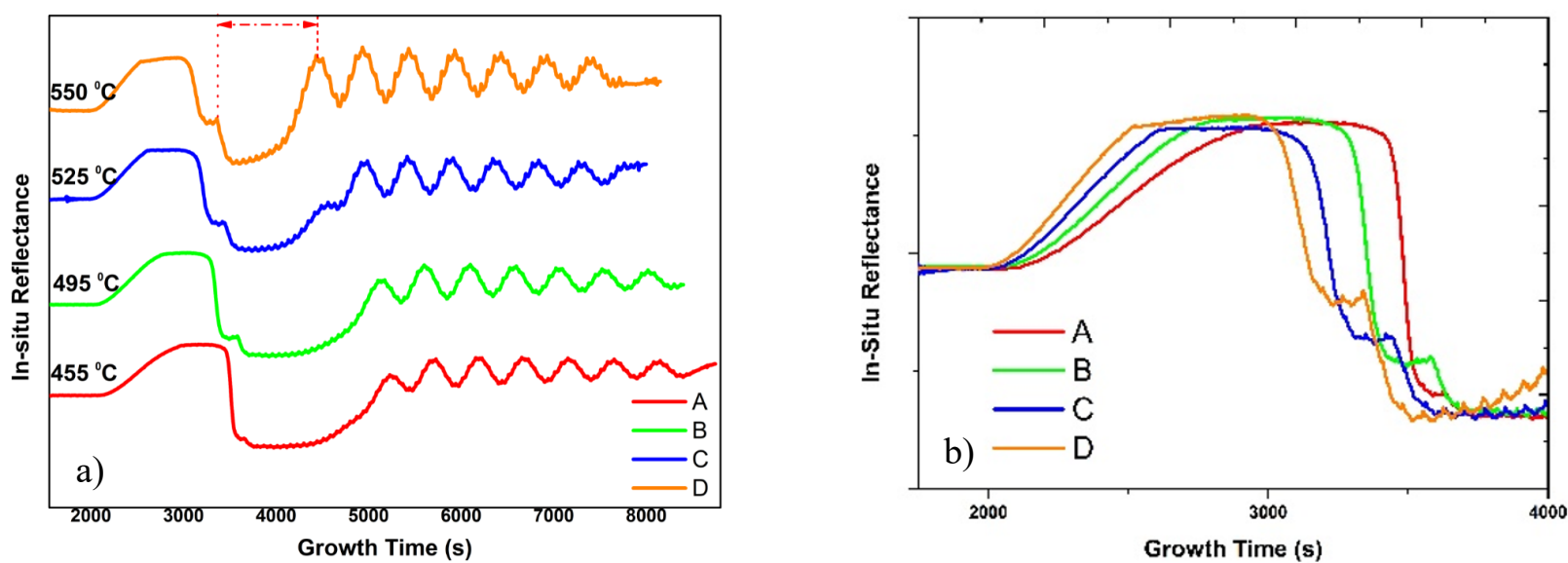

Figure 1. (a) reflectance measurements obtained during the growth for Sample A-D (b) comparison of the growth rate of nucleation layers with different LT-GaN growth temperatures.

islands occur with low temperature differences at the same annealing time. Lin Shang et al. have shown that size bigger islands have a fast recovery time [26].

HRXRD is a common technique to analyze the epitaxial layers for both rapid control and optimization of the used growth parameters [27]. The XRD measurement for (00.2) and (10.2) planes are done and their FWHM values are given information about the crystalline perfection. For this purpose, rocking curves of (00.2) and (10.2) planes in HRXRD measurement are obtained to investigate the influence of different LT-GaN growth temperatures on the crystal qualities of subsequent GaN epilayer.

Figure 3. gives the change of FWHM values obtained from symmetrical and asymmetrical scans with increasing the growth temperature of the nucleation layer.

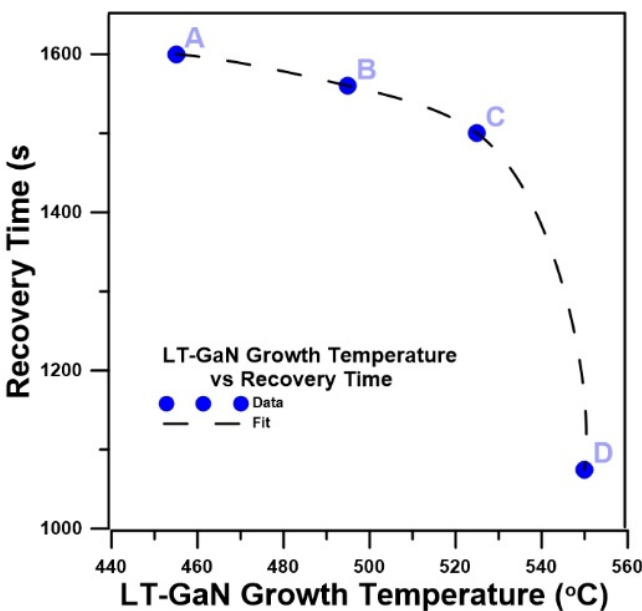

Figure 2. The variation of LT-GaN growth temperature versus recovery time.
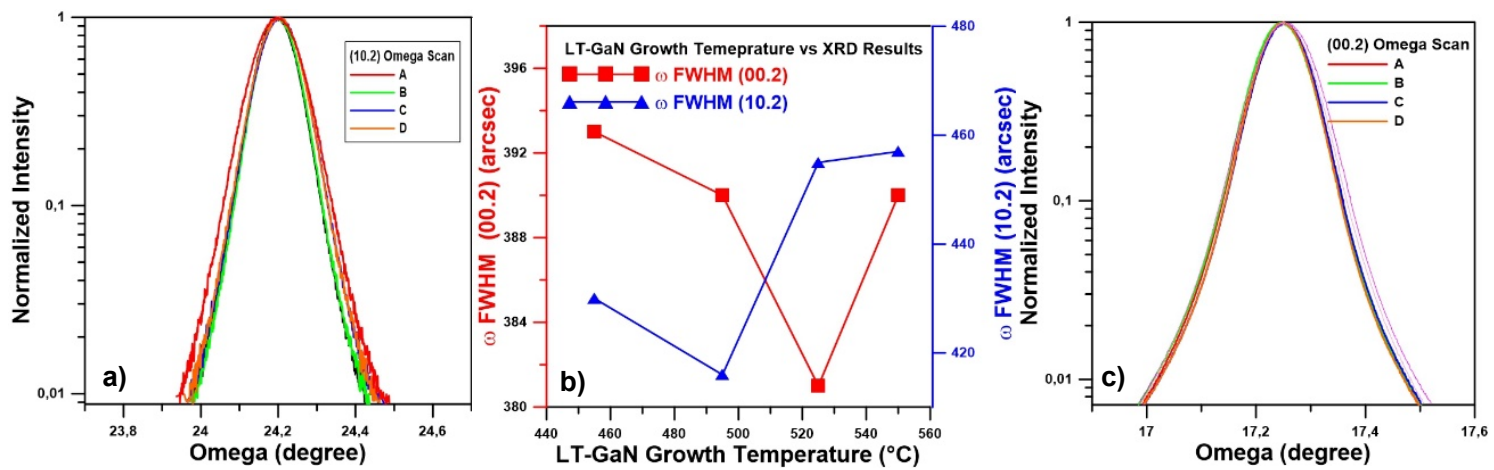

Figure 3. (a) symmetric, (b) asymmetric omega scans, and (c) the variation of FWHM values obtained by HRXRD for (00.2) and (10.2) planes with the increase of LT-GaN growth temperature

The FWHM of the (00.2) and (10.2) rocking curve spectra can be affected edge, screw, and mixed dislocations in the structure [28]. As shown in Figure 3, FWHM values obtained from reflections in (00.2) and (10.2) planes decrease from 393 to 390 arcsec and from 430 to 416 arcsec, respectively when LT-GaN growth temperature is increased from $455{ }^{0} \mathrm{C}$ to $495{ }^{0} \mathrm{C}$. The FWHM value of (10.2) rocking curve begins to increase while the decrease in FWHM value of (00.2) rocking curve continues when LT-GaN growth temperature is raised to $520{ }^{\circ} \mathrm{C}$. FWHM value of (00.2) rocking curve starts to increase when the LT-GaN growth temperature is increased to $550{ }^{0} \mathrm{C}$. It is observed that the LT-GaN growth temperature does not have a significant effect on the FWHM values obtained from the rocking curves of the $(00.2)$ plane. The increased ratio $\left({ }^{0} \mathrm{C} / \mathrm{s}\right)$ in temperature during annealing is different though all samples have the same annealing time. L. Sugiura et al. have analyzed the influence of annealing time (ramping rate of temperature) on subsequent GaN layers. They have observed a similar tendency in the chancing of FWHM values comparing with our study. They have found that the fast temperature ramping between LT-GaN and HT-GaN growths produces the subsequent $\mathrm{GaN}$ epilayers with both rough surfaces and worse quality [29]. Generally, dislocations that occur at the boundaries of nucleation islands are edge type while dislocations that come from inside the nucleation islands are screw/mixed type [30]. It is thought 
that when LT-GaN growth temperature is altered, the sizes of islands change because of different transition times for each sample. According to HRXRD measurement, the ideal growth conditions can be determined with the changing of LT-GaN growth temperature in terms of sizes of nucleation islands.

PL measurement which is the most common, and nondestructive is a characterization device to investigate the optical properties of structures [31]. Figure 4 demonstrates PL spectra $(310-680 \mathrm{~nm})$ obtained at room temperature for Sample A-D. The PL spectra for all samples include two main peaks; (1) high intensity and narrow PL band called near band edge luminescence at $\sim 361 \mathrm{~nm}$ and (2) low intensity and broad PL band called yellow luminescence at $\sim 550 \mathrm{~nm}$. In undoped $\mathrm{GaN}$, the band edge luminescence is occurred by the transition from the conduction band to valance band while yellow luminescence depends on point defects that come from impurity and growth conditions. Point defects have an important role in the optical and electrical properties of the $\mathrm{GaN}$ epilayer and related electronic and photonic devices. However, a majority of the point defects in the structure remain still unanswered [32].

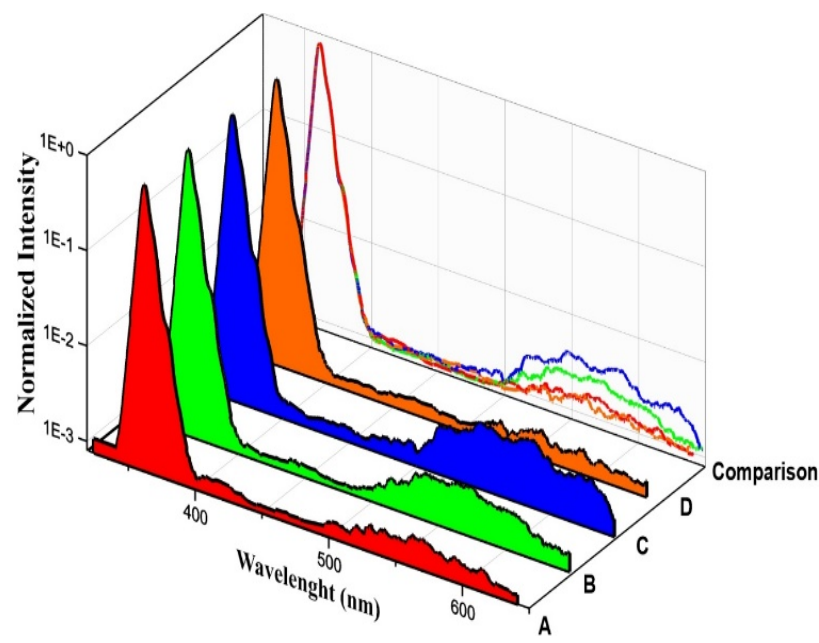

Figure 4. The behavior of photoluminescence spectra for samples with different LT-GaN growth temperatures.

Generally, the comparing intensity ratio between the near band edge peak and yellow luminescence peak is a common way to compare the quality of GaN films [33]. Therefore, the effect of LT-GaN growth temperature on the ratio of near band edge intensity to yellow band intensity is investigated. Figure 5 gives the comparing the ratio of band edge intensity to yellow luminescence intensity with changing LT-GaN growth temperature. It is clear that the intensity ratio changes with the variation of LT-GaN growth temperature which shows that the optical quality of $\mathrm{GaN}$ is related to the growth temperature of the nucleation layer. Near band edge intensities of all samples are normalized to see the effect of LT-GaN growth temperature on optical properties of subsequent $\mathrm{GaN}$ layer. The value of the intensity ratio changes with yellow luminescence intensity. It can be said that point defects for Sample B, C, and D may have increased with increasing LT-GaN growth temperature compared to Sample A because of their yellow luminescence intensities.

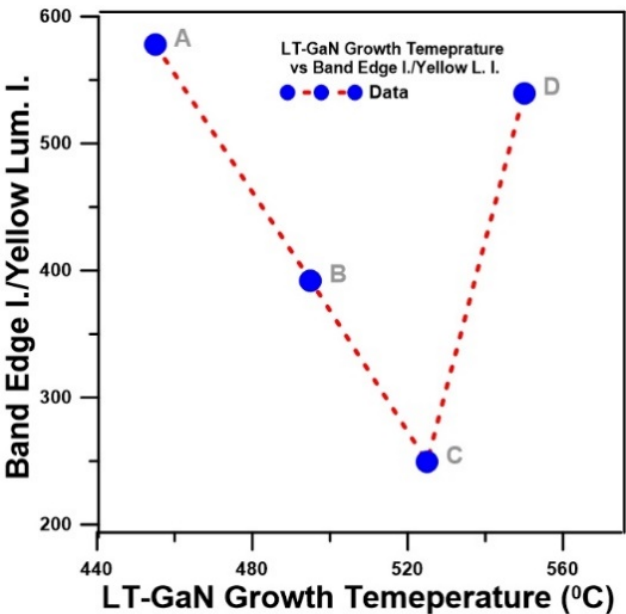

Figure 1. LT-GaN growth temperature versus the ratio of band edge intensity to yellow luminescence intensity.

\section{Conclusions}

In this study, the effect of LT-GaN growth temperature on structural and optical properties of subsequent GaN layer grown on a sapphire substrate using the MOVPE technique is investigated. In-situ reflectance curves show that transition time increases with increasing LT-GaN growth temperature because of the changing of GaN nucleation islands' sizes. The rocking curves of (00.2) and (10.2) planes in HRXRD measurement are obtained to show the effect of nucleation layer temperature on the crystal qualities of the HT-GaN epilayer. The results of HRXRD measurement demonstrate that there is an improvement in FWHM of both (00.2) and (10.2) rocking curves with changing of LT-GaN growth temperature. PL spectra for all samples include two main peaks which correspond to band edge and yellow luminescence. It is observed that the intensity of yellow luminescence caused by point defects changes with LT-GaN growth temperature.

\section{Acknowledgments}

The author acknowledges the usage of the Nanophotonics Research and Application Center at Cumhuriyet University (CUNAM) facilities. This study is supported by the TUBITAK under project nos. 113G103 and 115E109. The author thanks Ms. A. Alev Kizilbulut from ERMAKSAN Optoelectronics for PL measurements.

This study does not require ethics committee approval.

\section{References}

[1] Jain, S., Willander, M., Narayan, J., Overstraeten, R. V. (2000). III-nitrides: Growth, characterization, and properties, Journal of Applied Physics, 87(3):965-1006.

[2] Demir, I. (2018). Growth temperature dependency of high Al content $\mathrm{AlGaN}$ epilayers on $\mathrm{AlN} / \mathrm{Al} 2 \mathrm{O} 3$ templates, Cumhuriyet Science Journal, 39(3):728-733.

[3] Demir, I., Li, H., Robin, Y., McClintock, R., Elagoz, S., Razeghi, M. (2018). Sandwich method to grow high quality AlN by MOCVD, Journal of Physics D: Applied Physics, 51(8):085104. 
[4] Demir, I., Robin, Y., McClintock, R., Elagoz, S., Zekentes, K., Razeghi, M. (2017). Direct growth of thick AlN layers on nanopatterned $\mathrm{Si}$ substrates by cantilever epitaxy, Physica Status Solidi (a), 214(4):1600363.

[5] Fan, X., Xu, S., Li, P., Zhang, J., Peng, R., Zhao, Y., Du, J., Hao, Y. (2019). Nonpolar and semipolar ultraviolet multiple quantum wells on $\mathrm{GaN} /$ sapphire, Materials Science in Semiconductor Processing, 92:103-107.

[6] Genç, M., Sheremet, V., Altuntaş, I., Demir, I., Gür, E., Elagöz, S., Gülseren, O., Özgür, U., Avrutin, V., Morkoç, H. (2020). PECVD grown SiN photonic crystal microdomes for the light extraction enhancement of GaN LEDs, Gallium Nitride Materials and Devices $X V$, International Society for Optics and Photonics, $112800 \mathrm{O}$.

[7] Genç, M., Sheremet, V., Elçi, M., Kasapoğlu, A., Altuntaş, I., Demir, I, Eğin, G., Islamoğlu, S., Gür, E., Muzafferoğlu, N. (2019). Distributed contact flip chip InGaN/GaN blue LED; comparison with conventional LEDs, Superlattices and Microstructures, 128:9-13.

[8] Remesh, N., Kumar, S., Guiney, I., Humphreys, C. J., Raghavan, S., Muralidharan, R., Nath, D.,N. (2019). A Novel Technique to Investigate the Role of Traps in the OffState Performance of AlGaN/GaN High Electron Mobility Transistor on Si Using Substrate Bias, Physica Status Solidi (a), 217(7):1900794.

[9] Robin, Y., Ding, K., Demir, I., McClintock, R., Elagoz, S., Razeghi, M. (2019). High brightness ultraviolet lightemitting diodes grown on patterned silicon substrate, Materials Science in Semiconductor Processing, 90:87-91.

[10] Taya, P., Singh, V., Jana, D., Tyagi, R., Sharma, T. (2019).

Optical characterization of InAlN/AlN/InGaN/GaN/ sapphire high electron mobility transistor structures, AIP Conference Proceedings, AIP Publishing LLC, 030467.

[11] Toci, G., Gizzi, L. A., Koester, P., Baffigi, F., Fulgentini, L., Labate, L., Hospodkova, A., Jary, V., Nikl, M., Vannini, M. (2019). InGaN/GaN multiple quantum well for superfast scintillation application: Photoluminescence measurements of the picosecond rise time and excitation density effect, Journal of Luminescence, 208:119-124.

[12] Amano, H., Sawaki, N., Akasaki, I., Toyoda, Y. (1986). Metalorganic vapor phase epitaxial growth of a high quality GaN film using an AlN buffer layer, Applied Physics Letters, 48(5): 353-355.

[13] Anisimov, A., Wolfson, A., Mokhov, E. (2018). Raman Spectra of Thick Epitaxial GaN Layers Formed on SiC by the Sublimation Sandwich Method, Semiconductors, 52(9):1225-1227.

[14] Klad'ko, V. P., Chornen'kii, S.V., Naumov, A. V., Komarov, A. V., Tacano, M., Sveshnikov, Y. N., Vitusevich, S., Belyaev, A. E. (2006). Interface structural defects and photoluminescence properties of epitaxial GaN and $\mathrm{AlGaN} / \mathrm{GaN}$ layers grown on sapphire, Semiconductors, 40(9):1060-1065.

[15] Timoshnev, S., Mizerov, A., Sobolev, M., Nikitina, E. (2018). Growth of GaN Layers on Si (111) Substrates by Plasma-Assisted Molecular Beam Epitaxy, Semiconductors, 52(5):660-663.

[16] Mohammad, S. N., Salvador, A. A., Morkoc, H. (1995). Emerging gallium nitride based devices, Proceedings of the IEEE, 83(10):1306-1355.

[17] Altuntas, I. Demir, I., Kasapoğlu, A. E., Mobtakeri, S., Gür, E., Elagoz, S. (2017). The effects of two-stage HT-GaN growth with different V/III ratios during 3D-2D transition, Journal of Physics D: Applied Physics, 51(3):035105.

[18] Demir, I., Altuntas, I., Kasapoğlu, A., Mobtakeri, S., Guer, E., Elagoz, S. (2018). Microstructural evolution of MOVPE grown $\mathrm{GaN}$ by the carrier gas, Semiconductors, 52(16):2030-2038.
[19] Ito, T., Sumiya, M., Takano, Y., Ohtsuka, K., Fuke, S. (1999). Influence of thermal annealing on $\mathrm{GaN}$ buffer layers and the property of subsequent GaN layers grown by metalorganic chemical vapor deposition, Japanese Journal of Applied Physics, 38(2R):649.

[20] Kim, K. S., Oh, C. S., Lee, K. J., Yang, G. M., Hong, C. H., Lim, K. Y., Lee, H. J., Yoshikawa, A. (1999). Effects of growth rate of a $\mathrm{GaN}$ buffer layer on the properties of $\mathrm{GaN}$ on a sapphire substrate, Journal of Applied Physics, 85(12):8441-8444.

[21] Miyake, H., Motogaito, A., Hiramatsu, K. (1999). Effects of reactor pressure on epitaxial lateral overgrowth of GaN via low-pressure metalorganic vapor phase epitaxy, Japanese Journal of Applied Physics, 38(9A):L1000.

[22] Yi, M., Lee, H., Kim, D., Park, S., Noh, D., Kim, C., Je, J. (1999). Effects of growth temperature on $\mathrm{GaN}$ nucleation layers, Applied Physics Letters, 75(15):2187-2189.

[23] Koleske, D. D., Henry, R. L., Twigg, M. E., Culbertson, J. C., Binari, S. C., Wickenden, A. E., Fatemi, M. (2002). Influence of AlN nucleation layer temperature on $\mathrm{GaN}$ electronic properties grown on $\mathrm{SiC}$, Applied Physics Letters, 80(23):4372-4374.

[24] Nakamura, S. (1991). In situ monitoring of GaN growth using interference effects, Japanese Journal of Applied Physics, 30(8R):1620.

[25] Kim, S. , Oh, J., Kang, J., Kim, D., Won, J., Kim, J. W., Cho, H. K. (2004). Two-step growth of high quality GaN using V/III ratio variation in the initial growth stage, Journal of Crystal Growth, 262(1-4):7-13.

[26] Shang, L., Lu, T., Zhai, G., Jia, Z., Zhang, H., Ma, S., Li, T., Liang, J., Liu, X., Xu, B. (2015). The evolution of a $\mathrm{GaN} /$ sapphire interface with different nucleation layer thickness during two-step growth and its influence on the bulk GaN crystal quality, RSC Advances, 5(63):5120151207.

[27] Moram, M., Vickers, M. (2009). X-ray diffraction of IIInitrides, Reports on Progress in Physics, 72(3):036502.

[28] Heinke, H., Kirchner, V., Einfeldt, S., Hommel, D. (2000). $\mathrm{X}$-ray diffraction analysis of the defect structure in epitaxial GaN, Applied Physics Letters, 77(14):2145-2147.

[29] Sugiura, L., Itaya, K., Nishio, J., Fujimoto, H., Kokubun, Y. (1997). Effects of thermal treatment of low-temperature $\mathrm{GaN}$ buffer layers on the quality of subsequent GaN layers, Journal of Applied Physics, 82(10):4877-4882.

[30] Ning, X., Chien, F., Pirouz, P., Yang, J., Khan, M. A. (1996). Growth defects in GaN films on sapphire: The probable origin of threading dislocations, Journal of Materials Research, 11(3):580-592.

[31] Pittet, P., Lu, G. N., Galvan, J. M., Bluet, J. M., Anas, I., Giraud, J. Y., Balosso, J. (2009). PL characterization of GaN scintillator for radioluminescence-based dosimetry, Optical Materials, 31(10):1421-1424.

[32] Zhang, H., Reber, A. C., Geng, L., Rabayda, D., Wu, H., Luo, Z., Yao, J., Khanna, S. N. (2019). Formation of Al+ (C6H6) 13: The Origin of Magic Number in MetalBenzene Clusters Determined by the Nature of the Core, CCS Chemistry, 1(5):571-581.

[33] Robins, L. H., Bertness, K. A., Barker, J. M., Sanford, N. A., Schlager, J. B. (2007). Optical and structural study of GaN nanowires grown by catalyst-free molecular beam epitaxy. II. Sub-band-gap luminescence and electron irradiation effects, Journal of Applied Physics, 101(11):113506. 